\title{
Detection of Frederiksenia sp. isolated from a cat with nephritis - Short communication
}

\section{Acta Veterinaria Hungarica}

$68(2020) 2,140-146$

\section{DOI:}

$10.1556 / 004.2020 .00021$

() 2020 The Author(s)

\section{ORIGINAL ARTICLE}

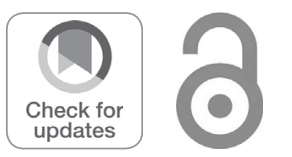

*Corresponding author. Tel.: +361467 4092 E-mail: magyar.tibor@agrar.mta.hu

\author{
BARBARA UJVÁRI ${ }^{1}$, LEVENTE SZEREDI ${ }^{2}$ and \\ TIBOR MAGYAR ${ }^{1 *}$ ๑ \\ ${ }^{1}$ Institute for Veterinary Medical Research, Centre for Agricultural Research, P.O. Box 18, Budapest, \\ H-1581 Hungary \\ ${ }^{2}$ Veterinary Diagnostic Directorate, National Food Chain Safety Office, Budapest, Hungary
}

Received: January 13, 2020 • Accepted: March 24, 2020

Published online: September 4, 2020

\section{KEYWORDS}

Frederiksenia, Pasteurellaceae, phylogeny, antimicrobial susceptibility, MIC

The family Pasteurellaceae is a large bacterial family that comprises 30 genera with validly published names (Christensen and Bisgaard, 2018; Dickerman et al., 2020). Bacteria belonging to the family Pasteurellaceae are commonly isolated from various animal species and humans; most are considered opportunistic pathogens (Olsen et al., 2005). Several newly described genera have recently been added to the family, including the most recently classified Caviibacterium and Conservatibacter, which are associated mainly with guinea pigs (Adhikary et al., 2018), and Glaesserella established by the reclassification of Haemophilus parasuis (Dickerman et al., 2020). These are expected to be supplemented with further new genera and species in the future.

Members of the family Pasteurellaceae have frequently been isolated from the oral cavity and upper respiratory tract of cats and dogs (Christensen and Bisgaard, 2008), including [Haemophilus] felis, Frederiksenia canicola and species of Pasteurella sensu stricto (Pasteurella multocida, Pasteurella dagmatis, Pasteurella canis, Pasteurella stomatis and Pasteurella oralis) (Mutters et al., 1985; Inzana et al., 1992; Korczak et al., 2014). Recently, P. dagmatis-like organisms have also been identified (Sellyei et al., 2010) from the oral cavity of cats. These species often cause bite wound infections in humans (Mutters et al., 1985).

Acquiring knowledge of newly isolated species is essential for epidemiological studies and routine diagnostic clinical microbiology laboratories. The aim of this study was to characterise a phenotypically and phylogenetically distinct strain, isolated from a diseased cat, that differed from the other members of Pasteurellaceae. 
In September 2018, a 4-month-old female Maine Coon cat suddenly died without apparent clinical signs and was submitted for routine postmortem examination to the NFCSO Veterinary Diagnostic Directorate (Budapest, Hungary). Tissue samples were collected from brain, heart, lungs, liver, spleen, kidney and mesenteric lymph nodes, and after fixation in $10 \%$ formalin they were processed for histological and immunohistochemical (IHC) examinations. Besides Giemsa staining, tissue sections were examined with an anti-Mycobacterium bovis-based IHC (Szeredi et al., 2008). This method detects several bacteria, fungi and parasites, and it is far more sensitive than traditional histochemical staining procedures, detecting even low numbers of the above-listed pathogens in tissue samples. Furthermore, tissue sections were immunolabelled with an anti-Bibersteinia trehalosi reagent, which shows cross-reaction with Mannheimia haemolytica and P. multocida types A, B and D by IHC (Szeredi et al., 2010). Samples from kidney and spleen were cultured on Columbia agar (LAB M Ltd., Bury, UK) supplemented with $5 \%$ sheep blood under aerobic conditions at $37{ }^{\circ} \mathrm{C}$ for $24 \mathrm{~h}$. Biochemical tests were performed for phenotypic characterisation according to the recommendation of Christensen et al. (2007).

Postmortem examination revealed severe icterus and slightly enlarged lungs, spleen and mesenteric lymph nodes presenting congestion. Kidneys were enlarged about $1.5 \times$ to the normal size, they were dark red in colour, and numerous greyish-white foci of $1 \mathrm{~mm}$ in diameter were dispersed in the cortex. The main histological lesion was observed in the kidneys presenting severe embolic suppurative nephritis. Large numbers of bacterial emboli occluded the glomerular capillaries. Numerous acute thrombi associated with large areas of necrosis and haemorrhages were also evident. Lesions indicative of bacterial septicaemia were detected in other organs as well: acute serous hepatitis accompanied by scattered foci of acute inflammation and necrosis, acute serous-purulent myocarditis with segmental or circumflex vascular necrosis, mild acute interstitial pneumonia, mild non-purulent encephalitis, and lymphoid depletion in the spleen. Large numbers of bacteria were detected in the glomerular capillaries of the kidney with Giemsa staining and by an anti-M. bovis-based IHC method. Similar bacteria were identified in the foci of acute necrotic inflammation in the liver, and in the sinusoids of the spleen. These bacteria were not immunolabelled by an anti-B. trehalosi reagent (Fig. 1).

From the kidney and the spleen, Pasteurella-like bacterial colonies were grown in pure culture, which we designated strain 6036. Strain 6036 was non-haemolytic and after $24 \mathrm{~h}$ of aerobic incubation on Columbia agar plates the colonies were $0.5-1 \mathrm{~mm}$ in diameter with regular circular shape. They did not adhere to the agar surface. Their surface was smooth, shiny and greyish. The cells were Gram-negative, non-motile rods. $\mathrm{V}$ - or X-factor dependence was not observed and strain 6036 did not grow on MacConkey agar. Strain 6036 was oxidase, catalase and indole positive, but negative for ornithine decarboxylase and urea. Acid was produced from glucose, maltose and xylose, but not from trehalose, lactose, arabinose, dulcitol and sorbitol.

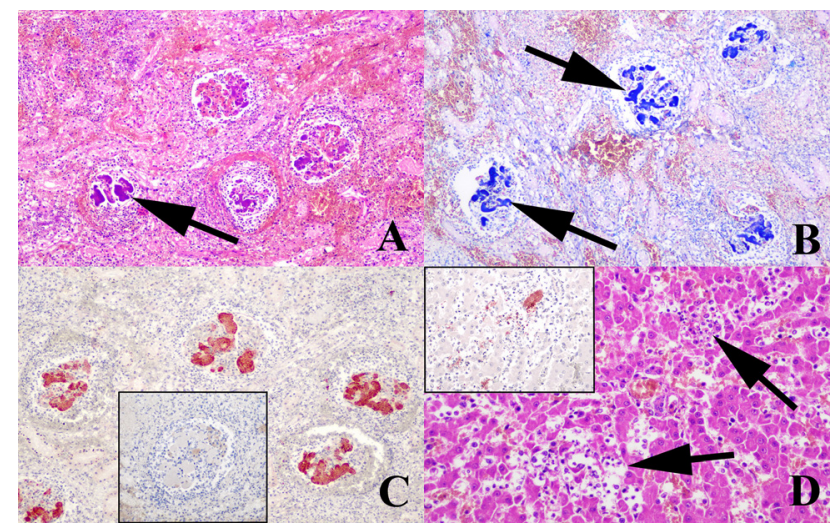

Fig. 1. A-C. Kidney, cat. A: Severe embolic (arrow) glomerulonephritis associated with acute haemorrhages and necrosis. Haematoxylin and eosin $(\mathrm{HE}), \times 100$; B: Several bacterial emboli (arrows) in the capillaries of glomeruli. Giemsa, $\times 100$; C: Strong immunostaining of bacterial emboli. Anti-Mycobacterium bovis based IHC, $\times 100$; Inset: Bacterial emboli are not immunostained with reagent specific for B. trehalosi, M. haemolytica and P. multocida A, B and D. IHC, $\times 200$; D: Liver, cat. Acute serous hepatitis associated with foci of acute inflammation and necrosis (arrows). HE, $\times 200$; Inset: Large amount of bacteria are immunostained in the area of inflammatory infiltration and in focal necrosis. Anti-M. bovis based IHC, $\times 200$

Phylogenetic analysis was performed by sequencing segments of their 16S rRNA, rpoB (encoding the beta subunit of the RNA polymerase), recN (encoding the DNA repair protein) and infB (encoding translation initiation factor 2) genes as described previously (Korczak et al., 2014). Sequencing of PCR products was performed by Macrogen Europe (Amsterdam, The Netherlands). Nucleotide sequences were aligned and compared using Geneious Prime software (version 2019.2.1; http://www.geneious.com). The GenBank accession numbers for sequences obtained in this study are MN701139-MN701142. Nucleotide sequence data were analysed using MEGA7 software (Kumar et al., 2016). The evolutionary history was inferred using the neighbourjoining model and the dataset was subjected to bootstrap analysis of 1000 replicates. Multilocus sequence analysis (MLSA) was also performed (Korczak et al., 2014). Sequence identity data of the four genes were calculated using the Geneious Prime program and used in the formula described by Zeigler (2003):

$$
\text { SI genome }=-1.30+2.25(\operatorname{SIrec} N),
$$

where SI is sequence identity.

Phylogenetic trees based on the alignment of the 16S rRNA gene sequences (Fig. 2) and MLSA of the four concatenated genes confirmed the relatedness of strain 6036 to the Pasteurellaceae family and placed it on a species-like branch (Fig. 3). The 16S rRNA gene sequence comparison showed the highest sequence identity with the type strain of F. canicola (95.14\%). Based on $r p o B$ sequence identity, strain 6036 showed the highest identity with $B$. trehalosi (90.58\%). Inf $B$-based phylogeny showed the closest relatedness of 


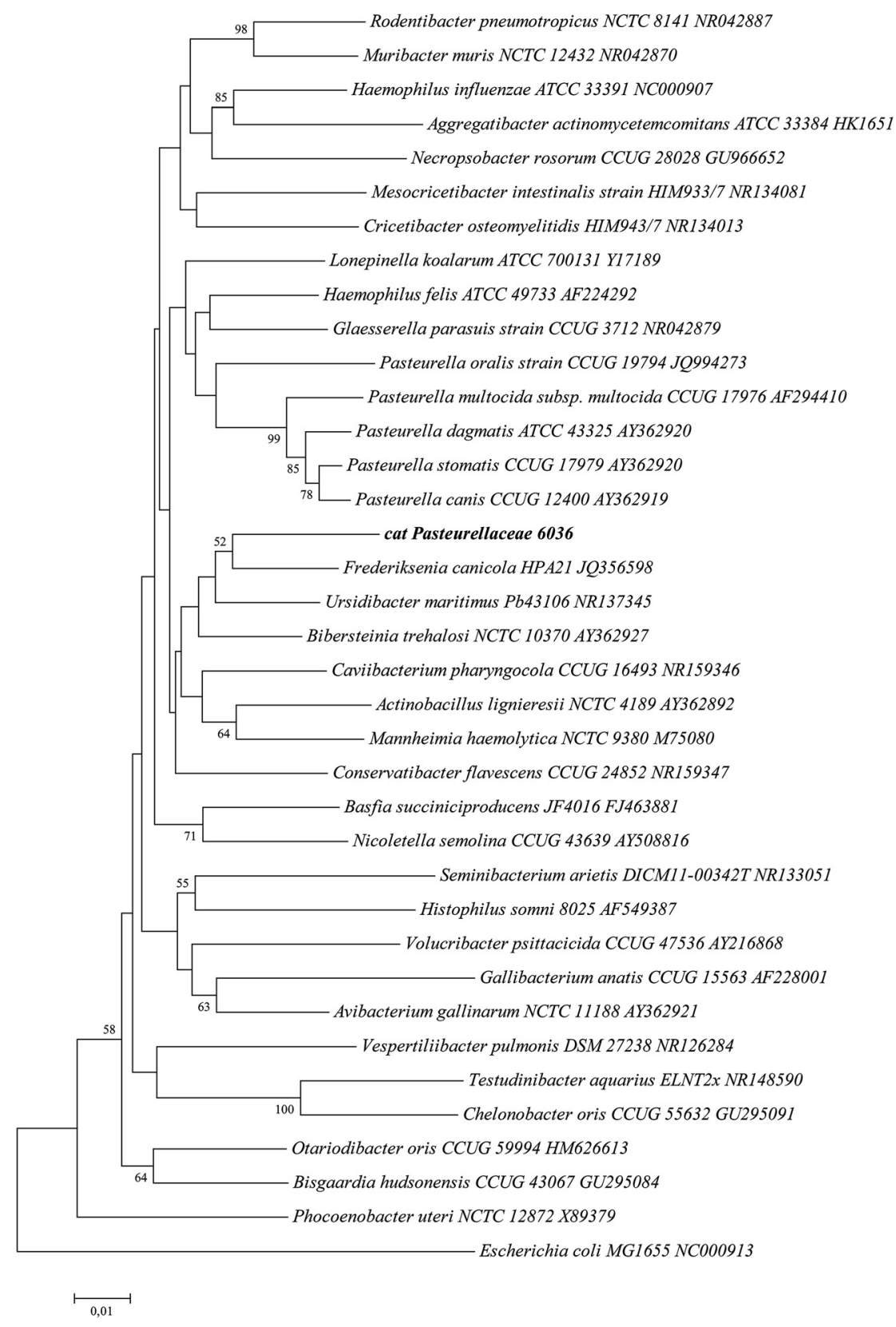

Fig. 2. Phylogenetic relationship of strain 6036 and other members of the family Pasteurellaceae. The evolutionary history was inferred using the neighbour-joining method based on partial $16 \mathrm{~S}$ rRNA gene sequences. Escherichia coli was included as an outgroup to root the dendrogram

strain 6036 with [Haemophilus] felis ATCC 49733 (89.50\%); the $\operatorname{recN}$ sequence identity was the highest with the type species of the Frederiksenia genus $(76.88 \%)$ again. Lower genetic relatedness was detected when comparing the $16 \mathrm{~S}$ rRNA (91.28-92.43\%), rpoB (84.37-86.37\%), infB (78.86$82.43 \%)$ and $\operatorname{recN}(59.55-61.11 \%)$ gene sequences with species representing the genus Pasteurella. Sequence data confirmed that strain 6036 is reliably identifiable using DNA sequence-based approaches.

Calculation of whole-genome relatedness by the presented $\operatorname{recN}$ sequence-based method is a reliable application for cross-comparison of Pasteurellaceae species and serves as an alternative to the classical hybridisation method (Zeigler,
2003, 2005; Kuhnert and Korczak, 2006; Christensen et al., 2012). Genome similarity values calculated for strain 6036 are listed in Table 1. Comparison of predicted genome similarity of strain 6036 and genera of Pasteurellaceae revealed that the similarity of $F$. canicola (strain HPA 21) and strain 6036 was 0.43 , which is above the threshold of 0.4 for genus separation (Kuhnert and Korczak, 2006). However, this value is below the threshold of 0.85 for species separation, which indicates that strain 6036 can be classified to the Frederiksenia genus but represents a species different from F. canicola.

A PCR based on the recN gene sequence was developed for identification and discrimination from other species. 


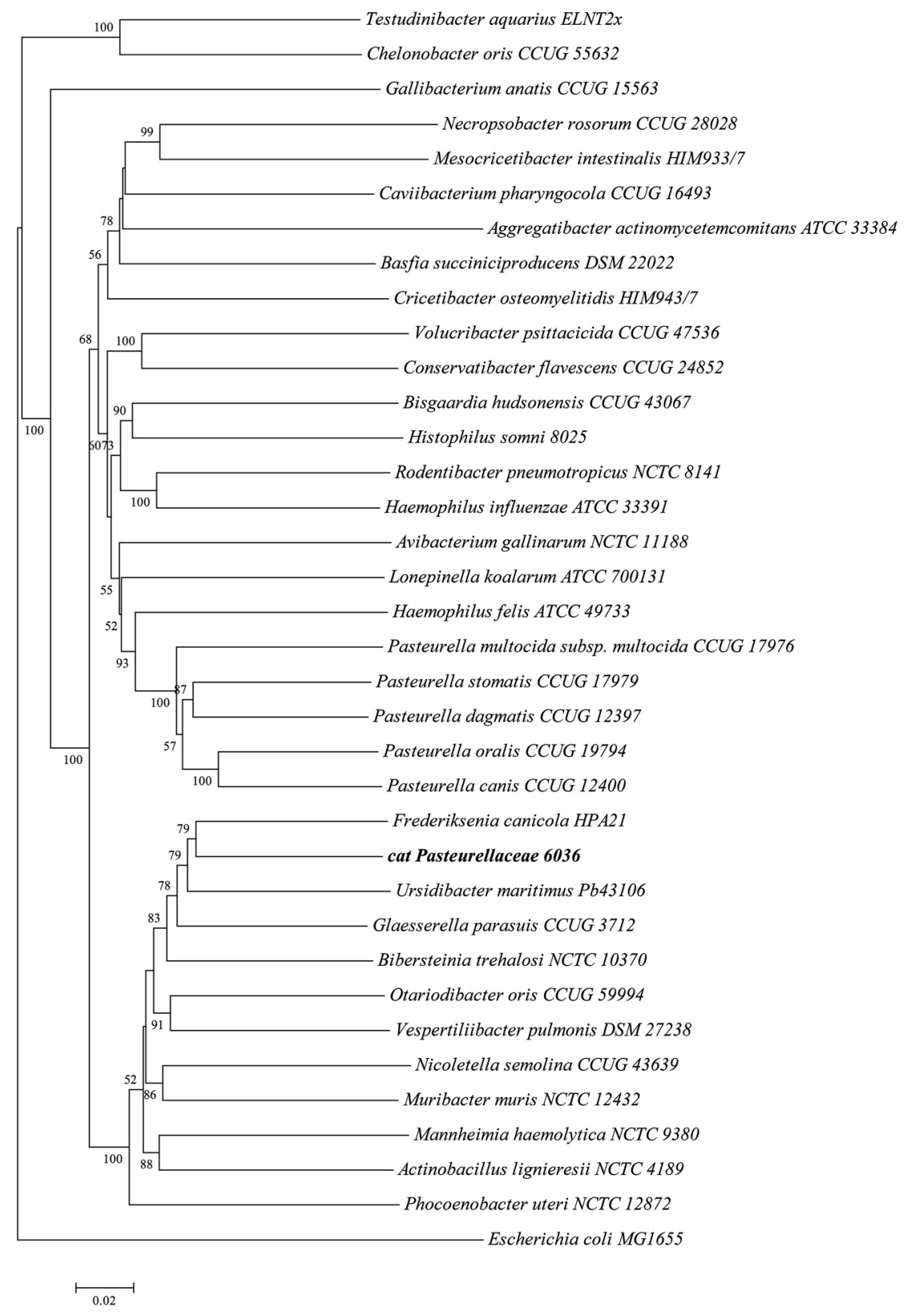

Fig. 3. Phylogenetic relationship of strain 6036 and other members of the family Pasteurellaceae. The evolutionary history was inferred using the neighbour-joining method based on concatenated $16 \mathrm{~S}$ rRNA gene, rpoB, recN and infB sequences. Escherichia coli was included as an outgroup to root the dendrogram

Primers 6036-F TTTGAATGGCTCAAACAGC and 6036-R CGGCAAGTTCTACACAACG, amplifying an 862-bp fragment, were used in the reaction. The reaction was complemented using primers PasrpoB-L and RpoB-R amplifying a 560-bp fragment of the rpoB gene (Korczak et al., 2004). Thus, by conducting a multiplex PCR we were able to exclude the possibility of a negative reaction due to PCR inhibition or lack of enough DNA. PCR was performed in a total volume of $25 \mu \mathrm{l}$ containing $2.5 \mu \mathrm{l}$ of $10 \times$ DreamTaq buffer, $0.5 \mathrm{mM}$ $\mathrm{MgCl}_{2}, 200 \mu \mathrm{M}$ dNTPs, $0.5 \mu \mathrm{M}$ of each primer and $1 \mathrm{U}$ of DreamTaq polymerase (Thermo Scientific, Waltham, USA). Reactions were run in a Bio-Rad C1000 Touch thermal cycler. DNA was amplified for 30 cycles using the following settings: denaturation at $95^{\circ} \mathrm{C}$ for $30 \mathrm{~s}$, annealing at $60^{\circ} \mathrm{C}$ for $30 \mathrm{~s}$ and extension at $72{ }^{\circ} \mathrm{C}$ for $1 \mathrm{~min}$. An initial denaturation step $\left(95^{\circ} \mathrm{C}, 10 \mathrm{~min}\right)$ and a final extension step $\left(72^{\circ} \mathrm{C}, 7 \mathrm{~min}\right)$ were also performed. Each reaction mixture was analysed using electrophoresis in a $1.5 \%$ agarose gel (SeaKem, Lonza, Basle, Switzerland) stained with GelRed (Biotium Inc., Hayward, USA) and visualised under UV light. Strain 6036 and species representing the family Pasteurellaceae were used to validate the PCR. The newly designed species-specific PCR clearly discriminated strain 6036 from other members of Pasteurellaceae. The PCR resulted in the expected amplification product with strain 6036 but did not produce any PCR product with other tested Pasteurellaceae species (Fig. 4). 
Table 1. Calculated genome similarity values of strain 6036 to type species of Pasteurellaceae genera based on recN sequences

\begin{tabular}{|c|c|c|c|}
\hline & Isolate 6036 & & $\begin{array}{c}\text { Isolate } \\
6036\end{array}$ \\
\hline Frederiksenia canicola HPA 21 & 0.43 & Cricetibacter osteomyelitidis HIM943/7 & 0.07 \\
\hline Vespertilibacter pulmonis DSM 27238 & 0.39 & $\begin{array}{c}\text { Caviibacterium pharyngocola CCUG } \\
16493\end{array}$ & 0.06 \\
\hline Ursidibacter maritimus $\mathrm{Pb} 43106$ & 0.38 & Basfia succiniproducens DSM22022 & 0.06 \\
\hline Muribacter muris NCTC 12432 & 0.38 & [Haemophilus] felis ATCC 49733 & 0.06 \\
\hline Otariodibacter oris CCUG 59994 & 0.37 & $\begin{array}{l}\text { Pasteurella multocida subsp. multocida } \\
\text { CCUG } 17976\end{array}$ & 0.06 \\
\hline Bibersteinia trehalosi NCTC 10370 & 0.36 & Pasteurella dagmatis ATCC 43325 & 0.05 \\
\hline Glaesserella parasuis CCUG 3712 & 0.35 & Bisgaardia hudsonensis CCUG 43067 & 0.05 \\
\hline Nicoletella semolina CCUG 43639 & 0.35 & Lonepinella koalarum ATCC 700131 & 0.05 \\
\hline Actinobacillus lignieresii NCTC 4189 & 0.32 & Pasteurella canis CCUG 12400 & 0.04 \\
\hline Mannheimia haemolytica NCTC 9380 & 0.28 & Pasteurella oralis CCUG 19794 & 0.04 \\
\hline Phocoenobacter uteri NCTC 12872 & 0.27 & $\begin{array}{c}\text { Rodentibacter pneumotropicus NCTC } \\
8141\end{array}$ & 0.04 \\
\hline Avibacterium gallinarum NCTC 11188 & 0.11 & Mesocricetibacter intestinalis HIM933/7 & 0.02 \\
\hline Volucribacter psittacicida CCUG 47536 & 0.10 & Necropsobacter rosorum CCUG 28028 & 0.02 \\
\hline $\begin{array}{l}\text { Conservatibacter flavescens CCUG } \\
24852\end{array}$ & 0.10 & Histophilus somni HS8025 & 0.01 \\
\hline Haemophilus influenzae ATCC 33391 & 0.09 & Testudinibacter aquarius ELNT2x & 0.01 \\
\hline Pasteurella stomatis CCUG 17979 & 0.07 & Chelonobacter oris CCUG 55632 & 0.00 \\
\hline $\begin{array}{l}\text { Aggregatibacter actinomycetemcomitans } \\
\text { ATCC } 33384\end{array}$ & 0.07 & Gallibacterium anatis CCUG 15563 & 0.00 \\
\hline
\end{tabular}

Antimicrobial susceptibility was tested to 18 antimicrobial agents using minimal inhibitory concentration (MIC) test strips according to the manufacturer's instructions (Liofilchem, Roseto, Italy; Table 2). Escherichia coli ATCC 25922 served as a quality control strain. MIC values were read after 24 -h incubation at $37^{\circ} \mathrm{C}$ and were defined as the lowest concentration with complete inhibitory action

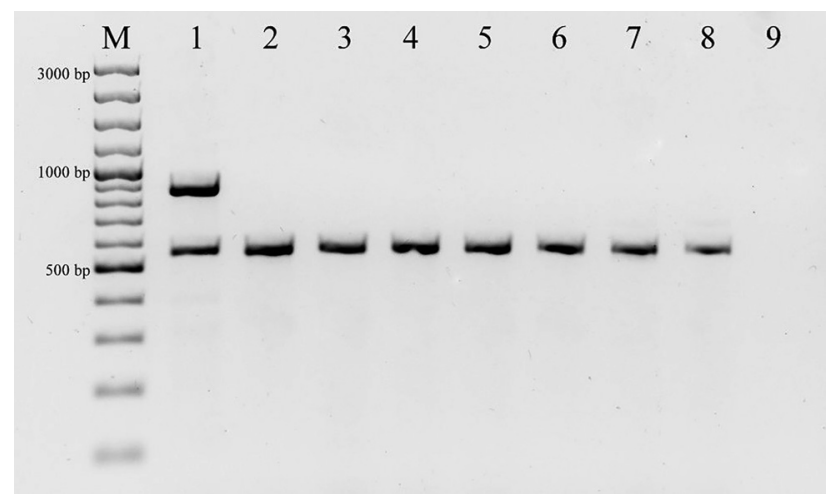

Fig. 4. Multiplex PCR resulting in an 862-bp fragment exclusively with strain 6036, and a positive signal for all investigated strains amplified with primers PasrpoB-L and RpoB-R, respectively. Lane M: molecular weight standard; lane 1: strain 6036; lane 2: Freder-

iksenia canicola HPA21; lane 3: Pasteurella multocida CCUG 17976; lane 4: Pasteurella dagmatis CCUG 12397; lane 5: Pasteurella canis CCUG 12400; lane 6: Rodentibacter pneumotropicus NCTC 8141; lane 7: Mannheimia haemolytica NCTC 9380; lane 8: Bibersteinia trehalosi NCTC 10370; lane 9: negative control according to the CLSI standards and as described in a previous study (CLSI, 2018a; CLSI, 2018b; Gutman et al., 2016).

Very limited information is available on the antibiotic susceptibility of F. canicola, and even this is on isolates from Tasmanian devils (Gutman et al., 2016). Frederiksenia is a relatively recently described genus (Korczak et al., 2014), so there are no breakpoints for antibiotic resistance testing. It is

Table 2. Minimum inhibitory concentrations (MIC) of antimicrobial agents for isolate 6036

\begin{tabular}{lc}
\hline Antimicrobial agent & $\begin{array}{r}\text { MIC for isolate } 6036 \\
(\mu \mathrm{g} / \mathrm{ml})\end{array}$ \\
\hline Penicillin G & 0.023 \\
Ampicillin & 0.047 \\
Streptomycin & 96 \\
Gentamicin & 8 \\
Tetracycline & 0.38 \\
Doxycycline & 0.38 \\
Erythromycin & 0.75 \\
Clindamycin & 2 \\
Enrofloxacin & 0.006 \\
Ciprofloxacin & 0.012 \\
Nalidixic acid & 0.75 \\
Chloramphenicol & 0.75 \\
Florfenicol & 0.5 \\
Cefazolin & 0.19 \\
Cefpodoxime & 0.016 \\
Colistin & 0.5 \\
Sulphamethoxazole & 16 \\
Trimethoprim - sulphamethoxazole & 0.19 \\
\hline
\end{tabular}


therefore not possible to determine unequivocally whether an isolate is susceptible or resistant to a given antibiotic, but it can be predicted from the exact MIC values. Low MIC values were determined for most of the antimicrobials tested (Table 2), including penicillin, ampicillin, tetracycline, doxycycline, erythromycin, enrofloxacin, ciprofloxacin, nalidixic acid, chloramphenicol, florfenicol, cefazolin, cefpodoxime, colistin, sulphamethoxazole and trimethoprim/sulphamethoxazole. Increased MIC values were obtained for streptomycin $(96 \mu \mathrm{g} / \mathrm{mL})$, gentamicin $(8 \mu \mathrm{g} / \mathrm{mL})$ and clindamycin $(2$ $\mu \mathrm{g} / \mathrm{mL})$. The MIC value for sulphamethoxazole $(16 \mu \mathrm{g} / \mathrm{mL})$ was not considered to be elevated because pathogens of veterinary importance can only be considered resistant to sulphonamides at an MIC equal to or greater than $512 \mu \mathrm{g} / \mathrm{mL}$ (CLSI, 2018a, 2018b). Aminoglycoside resistance occurs widely in Gram-negative bacteria, usually because of enzymatic inactivation of the drug (Kehrenberg et al., 2005; Giguére, 2013). Our results confirm the findings of Gutman et al. (2016), who found that resistance occurred intrinsically for aminoglycosides and lincosamides in bacterial species belonging to the family Pasteurellaceae. This highlights that avoiding the use of the aforementioned antibiotics can enable the application of appropriate therapeutic treatments and prevent the spread of antibiotic resistance.

The bacterium examined in the study was highly pathogenic for this kitten, causing severe glomerulonephritis and septicaemia. In the kidney and liver, the presence of bacteria was demonstrated by both Giemsa staining and an anti- $M$. bovis-based IHC method. The Pasteurella-like bacterium was antigenically clearly differentiated from $B$. trehalosi, M. haemolytica, P. multocida types A, B and D by the IHC test. Results of phenotypic and genetic analyses suggest that strain 6036 represents a novel species of the Frederiksenia genus, which is supported by individual and combined gene sequence analysis of the 16S rRNA gene and three housekeeping genes. Calculation of whole-genome relatedness with the $\operatorname{recN}$ sequence also confirmed this finding. This genus currently includes only the species F. canicola and, compared with strain 6036, we found differences in both genetic and phenotypic characteristics. The biochemical characteristics of strain 6036 show that xylose can be used for its phenotypic differentiation from F. canicola, which is a xylose-negative species (Korczak et al., 2014). We also designed a speciesspecific PCR that allows rapid and accurate identification and differentiation from $F$. canicola. In summary, new species may be added to the Frederiksenia genus in the future; in this report, we identified a possibly novel Frederiksenia-like organism by a combination of phenotypic and genetic analyses.

\section{ACKNOWLEDGEMENTS}

This work was supported by the National Research, Development and Innovation Fund, Hungary - Grant NKFIH K124457.

\section{REFERENCES}

Adhikary, S., Bisgaard, M., Nicklas, W. and Christensen, H. (2018): Reclassification of Bisgaard taxon 5 as Caviibacterium pharyngocola gen. nov., sp. nov. and Bisgaard taxon 7 as Conservatibacter flavescens gen. nov., sp. nov. Int. J. Syst. Evol. Microbiol. 68, 643-650.

Christensen, H. and Bisgaard, M. (2008): Taxonomy and biodiversity of members of Pasteurellaceae. In: Kuhnert, P. and Christensen, H. (eds.) Pasteurellaceae: Biology, Genomics and Molecular Aspects. Caister Academic Press, Norfolk. pp. 1-26.

Christensen, H. and Bisgaard, M. (2018): Classification of genera of Pasteurellaceae using conserved predicted protein sequences. Int. J. Syst. Evol. Microbiol. 68, 2692-2696.

Christensen, H., Bertelsen, M. F., Bojesen, A. M. and Bisgaard, M. (2012): Classification of Pasteurella species B as Pasteurella oralis sp. nov. Int. J. Syst. Evol. Microbiol. 62, 1396-1401.

Christensen, H., Kuhnert, P., Busse, H. J., Frederiksen, W. C. and Bisgaard, M. (2007): Proposed minimal standards for the description of genera, species and subspecies of the Pasteurellaceae. Int. J. Syst. Evol. Microbiol. 57, 166-178.

Clinical and Laboratory Standards Institute, CLSI (2018a): Performance Standards for Antimicrobial Disk and Dilution Susceptibility Tests for Bacteria Isolated from Animals, 4th ed. CLSI supplement VET08. CLSI, Wayne, PA.

Clinical and Laboratory Standards Institute, CLSI (2018b): Performance Standards for Antimicrobial Susceptibility Testing, 28th ed. CLSI supplement M100, Wayne, PA.

Dickerman, A., Aloka B. B. and Thomas, J. I. (2020): Phylogenomic analysis of Haemophilus parasuis and proposed reclassification to Glaesserella parasuis, gen. nov., comb. nov. Int. J. Syst. Evol. Microbiol. 70, 180-186.

Giguère, S. (2013): Lincosamides, pleuromutilins, and streptogramins. In: Giguère, S., Prescott, J. F. and Dowling, P. M. (eds.) Antimicrobial Therapy in Veterinary Medicine. Blackwell Publishing, Ames, Iowa. pp. 179-190.

Gutman, N., Hansen, M. J., Bertelsen, M. F. and Bojesen, A. M. (2016): Pasteurellaceae bacteria from the oral cavity of Tasmanian devils (Sarcophilus harrisii) show high minimum inhibitory concentration values towards aminoglycosides and clindamycin. Lett. Appl. Microbiol. 62, 237-242.

Inzana, T. J., Johnson, J. L., Shell, L., Møller, K. and Kilian, M. (1992): Isolation and characterization of a newly identified Haemophilus species from cats: Haemophilus felis. J. Clin. Microbiol. 30, 2108-2112.

Kehrenberg, C., Catry, B., Haesebrouck, F., de Kruif, A. and Schwarz, S. (2005): Novel spectinomycin/streptomycin resistance gene, aadA14, from Pasteurella multocida. Antimicrob. Agents Chemother. 49, 3046-3049.

Korczak, B. M., Bisgaard, M., Christensen, H. and Kuhnert, P. (2014): Frederiksenia canicola gen. nov., sp. nov. isolated from dogs and human dog-bite wounds. Antonie Leeuwenhoek 105, 731-741.

Korczak, B. M., Christensen, H., Emler, S., Frey, J. and Kuhnert, P. (2004): Phylogeny of the family Pasteurellaceae based on rpoB sequences. Int. J. Syst. Evol. Microbiol. 54, 1393-1399. 
Kuhnert, P. and Korczak, B. M. (2006): Prediction of wholegenome DNA-DNA similarity, determination of G + C content and phylogenetic analysis within the family Pasteurellaceae by multilocus sequence analysis (MLSA). Microbiol. 152, 25372548.

Kumar, S., Stecher, G. and Tamura, K. (2016): MEGA7: molecular evolutionary genetics analysis version 7.0 for bigger datasets. Mol. Biol. Evol. 33, 1870-1874.

Mutters, R., Ihm, P., Pohl, S., Frederiksen, W. and Mannheim, W. (1985): Reclassification of the genus Pasteurella Trevisan 1887 on the basis of deoxyribonucleic acid homology, with proposals for the new species Pasteurella dagmatis, Pasteurella canis, Pasteurella stomatis, Pasteurella anatis, and Pasteurella langaa. Int. J. Syst. Evol. Microbiol. 35, 309-322.

Olsen, I., Dewhirst, F. E., Paster, B. J. and Busse, H. J. (2005): Family Pasteurellaceae. In: Garrity, G. (ed.) Bergey's Manual of Systematic Bacteriology. Springer, New York. pp. 851-856.
Sellyei, B., Wehmann, E., Makrai, L. and Magyar, T. (2010): Characterisation of Pasteurella dagmatis-like isolates recovered from the feline oral cavity. Vet. Microbiol. 145, 279-285.

Szeredi, L., Glávits, R., Tenk, M. and Jánosi, S. (2008): Application of anti-BCG antibody for rapid immunohistochemical detection of bacteria, fungi and protozoa in formalin-fixed paraffinembedded tissue samples. Acta Vet. Hung. 56, 89-99.

Szeredi, L., Jánosi, S. and Pálfi, V. (2010): Microbiological and pathological examinations of fatal calf pneumonia cases induced by bacterial and viral respiratory pathogens. Acta Vet. Hung. 58, 341-356.

Zeigler, D. R. (2003): Gene sequences useful for predicting relatedness of whole genomes in bacteria. Int. J. Syst. Evol. Microbiol. 53, 1893-1900.

Zeigler, D. R. (2005): Application of a $\operatorname{recN}$ sequence similarity analysis to the identification of species within the bacterial genus Geobacillus. Int. J. Syst. Evol. Microbiol. 55, 1171-1179. 